
\title{
$\begin{array}{ll}\text { Research Square } & \text { Preprints are preliminary reports that have not undergone peer review. } \\ \text { They should not be considered conclusive, used to inform clinical practice, } \\ \text { or referenced by the media as validated information. }\end{array}$
}

\section{Elevated Average Maximum Intrabolus Pressure on High-Resolution Manometry is Associated with Esophageal Dysmotility and Delayed Esophageal Emptying on Timed Barium Esophagram}

Katelyn Madigan ( $\sim$ kmadigan@wakehealth.edu )

Wake Forest Baptist Medical Center

J. Shawn Smith

Prisma Health

Joni Evans

Wake Forest Baptist Medical Center

Steven Clayton

Wake Forest Baptist Medical Center

\section{Research Article}

Keywords: esophageal dysmotility, high-resolution manometry, timed barium esophagram

Posted Date: December 1st, 2021

DOI: https://doi.org/10.21203/rs.3.rs-1064649/v1

License: (1) (1) This work is licensed under a Creative Commons Attribution 4.0 International License.

Read Full License

Version of Record: A version of this preprint was published at BMC Gastroenterology on February 21st, 2022. See the published version at https://doi.org/10.1186/s12876-022-02165-5. 


\section{Abstract \\ Background}

Intrabolus pressure (IBP) recorded by high-resolution manometry (HRM) portrays the compartmentalized force on a bolus during esophageal peristalsis. HRM may be a reliable screening tool for esophageal dysmotility in patients with elevated average maximum IBP (AM-IBP). Timed barium esophagram (TBE) is a validated measure of esophageal emptying disorders, such as esophagogastric junction outflow obstruction and achalasia. This study aimed to determine if an elevated AM-IBP correlates with esophageal dysmotility on HRM and/or delayed esophageal emptying on TBE.

\section{Methods}

A retrospective analysis of all HRM (unweighted sample $n=155$ ) performed at a tertiary referral center from 09/2015-03/2017 yielded a case group $(n=114)$ with abnormal AM-IBP and a control group $(n=41)$ with a normal AM-IBP (pressure $<17 \mathrm{mmHg}$ ) as consistent with Chicago Classification 3 . All patients received a standardized TBE, with abnormalities classified as greater than $1 \mathrm{~cm}$ of retained residual liquid barium in the esophagus at 1 and 5 minutes or as tablet retention after 5 minutes.

\section{Results}

AM-IBP was significantly related to liquid barium retention $(p=0.003)$ and tablet arrest on timed barium esophagram ( $p=0.011)$. A logistic regression model correctly predicted tablet arrest in $63 \%$ of cases. Tablet arrest on AM-IBP correlated with an optimal prediction point at $20.1 \mathrm{mmHg}$ on HRM. Patients with elevated AM-IBP were more likely to have underlying esophageal dysmotility ( $95.6 \%$ vs. $70.7 \%$ respectively; $p<001)$, particularly esophagogastric junction outflow obstruction disorders. Elevated AMIBP was associated with incomplete liquid bolus transit on impedance analysis $(p=0.002)$.

\section{Conclusions}

Our findings demonstrate that an elevated AM-IBP is associated with abnormal TBE findings of esophageal tablet retention and/or bolus stasis. An abnormal AM-IBP (greater than $20.1 \mathrm{~mm} \mathrm{Hg}$ ) was associated with a higher probability of retaining liquid bolus or barium tablet arrest on TBE and esophageal dysmotility on HRM. This finding supports the recent incorporation of IBP in Chicago Classification v4.0.

\section{Introduction}

Esophageal bolus transit is a coordinated physiologic process in which esophageal smooth muscle contractility, intrabolus pressure (IBP), and deglutitive lower esophageal sphincter (LES) relaxation 
\{integrated relaxation pressure (IRP) \} determine the effectiveness of swallowing. ${ }^{1,2,3}$

IBP is the measurement of the compartmentalized pressure exerted on a solid or liquid bolus transiting through the esophagus, as shown in Figure 1. ${ }^{1-3}$ Elevated IBP can be an indirect indicator of esophageal obstruction. However, it is unknown if average maximum IBP (AM-IBP) correlates with esophageal dysmotility on HRM and delayed esophageal emptying on TBE, particularly in the advent of Chicago Classification version 4 (CCv4) in 2021.3,4 The previous conventional manometric equipment lacked the precision to measure dynamic IBP variations accurately. ${ }^{5-6}$, High-resolution manometry (HRM) provides a reliable screening tool for the initial detection of esophageal dysmotility. ${ }^{4,7}$ An increased number of recording sites with HRM creates a spatial continuum of intraluminal pressure through the interpolation between adjacent sensors. ${ }^{8,9}$ Timed barium esophagram (TBE) is a validated assessment to be used in conjunction with HRM for quantifying esophageal emptying in disorders causing esophageal outflow obstruction, such as EGJOO and achalasia. ${ }^{10-12}$

The calculation and clinical utility of AM-IBP was first described by Niebisch et al. in 2013. The AV-IBP is calculated $1 \mathrm{~cm}$ above the proximal border of the EGJ and displayed as the maximal average pressure of 3 nonconsecutive seconds. ${ }^{3-16}$ AM-IBP can be a surrogate marker for an obstructive process at the esophagogastric junction (EGJ). We hypothesized that AM-IBP would correspond to esophageal dysmotility on HRM and poor esophageal emptying on TBE. The objective of this study was to help fill an important gap in the literature by evaluating the relationship between elevated AM-IBP, esophageal dysmotility on HRM, and poor esophageal emptying on TBE.

\section{Materials And Methods}

\section{Study design and data source}

This was a retrospective cross-sectional analysis of all HRM performed at a tertiary referral center from September 2015 to March 2017.

\section{Study subjects}

Inclusion criteria included adult patients (ages 18 and over) for an unweighted sample size of $n=155$ patients with a referral for further clinical evaluation for non-obstructive dysphagia. We compared two groups: a case group of patients with an abnormal AM-IBP reading ( $n=114$ patients) and a control group with a normal AM-IBP reading ( $\mathrm{n}=41$ patients). Niebisch et al. described $14.6 \mathrm{mmHg}$ as the upper $95 \%$ value for healthy volunteers; therefore, we defined abnormal AM-IBP as IBP $>17 \mathrm{mmHg}$ in accordance with the ManoView ${ }^{\mathrm{TM}}$ software (Sierra Scientific Instruments Inc., Version 3.0, Los Angeles, CA, USA). ${ }^{16}$ All patients in both these groups also underwent a TBE study. In the initial unweighted sample of 157 patients, there were two patient exclusions to note due to intolerance: one patient did not receive measurements of the barium column, and another patient did not have measurements recorded at one minute. Further details of the specific study protocol are outlined below. 


\section{High-Resolution Manometry (HRM) Protocol}

We studied the esophageal motor function of all patients with the same 36-channel solid-state catheter system (Medtronic, Inc., Minneapolis, MN, USA) with high fidelity circumferential sensors at $1 \mathrm{~cm}$ intervals, as well as intra-luminal impedance. The manometry catheter with five intragastric sensors was placed trans-nasally and positioned to record from the hypopharynx to the stomach. After confirming patients had performed at least 6 hours of fasting before the test, patients were placed in the supine position. The protocol included a 3-minute baseline period and at least ten 5-mL water swallows, each separated by 30 -second intervals. Pertinent metric data recorded includes lower esophageal sphincter basal pressure, intrabolus pressure (IBP), integrated relaxation pressure (IRP), distal contractile latency (DCL), large breaks, frontal contractile velocity, and distal latency (DL). All data were analyzed using ManoView version 3.0 software in the high-resolution esophageal color topography mode for standardized data analysis. Studies were interpreted according to Chicago Classification version 3.0. In addition, to the IRP, basal LES sphincter pressure was recorded and considered abnormal if outside the accepted normative values (13 mmHg-43 mmHg). Earlier versions of ManoView did routinely report AMIBP. The AM-IBP was expressed as the average pressure $>2 \mathrm{mmHg}$ during deglutitive relaxation. AM-IBP is also measured relative to gastric pressure and calculated as the mean of the maximum pressure of three non-continuous seconds. Additionally, water-perfused HRM systems are available but were not used in this study. ${ }^{19}$

\section{Timed Barium Esophagram (TBE) Protocol}

TBE is a standardized protocol with the administration of $240 \mathrm{~mL}(8 \mathrm{oz})$ of low-density barium while the patient is standing upright. We obtained spot films at 1 minute and 5 minutes to assess liquid emptying from the esophagus. Barium column height was measured from the gastroesophageal (GE) junction to the height of the top of the column, with abnormal tests being the retention of liquid at 1 minute and five minutes. Following this measurement, the patient cleared their esophagus with water before ingesting a $13-\mathrm{mm}$ barium tablet. The barium height and width in centimeters were recorded at 1 minute and 5 minutes, with an abnormal test defined as the retention of the tablet at 5 minutes.

\section{Statistical Analysis}

Statistical analysis was performed using Microsoft Excel (version 1809). Continuous data were expressed as means with standard deviations, and we made comparisons with the Student's T-test. Discrete data were recorded as proportions, and we made comparisons using Chi-square tests. Two-sided p-values of 0.05 or less were considered significant. Additional statistical analysis was performed with logistic regression to evaluate the associations between AM-IBP and tablet arrest or barium height.

The following variables were included in a multivariable logistic regression model to help predict variables associated with abnormal TBE: BMI, Mean LES RBP, mean LES RP, LES IBP AM-IBP, Hypertensive LES, Type I-III Achalasia, EGJOO, combined other dysmotility (jackhammer esophagus, 
ineffective esophageal motility, diffuse esophageal spasm, hypotensive LES and pseudoachalasia), hiatal hernia and TBE tablet arrest.

\section{Results}

\section{Baseline characteristics}

Of the 155 patients tested between September 2015 and March 2017, the vast majority (114 patients) were found to have abnormal AM-IBP on HRM. There were 41 patients with normal AM-IBP in the control group, as shown in Table 1. The population's average age was 58 years \pm 13.2 years with an average BMI of $30.4 \pm 6.87$; there were no significant differences in age or BMI between the groups. Significantly more females were in the control group compared to the case group ( $80.5 \%$ vs. $58.8 \% ; p=0.01)$. As outlined in Table 2, there was significantly more abnormal manometry testing in the case group than in the control group $(95.6 \%$ vs. $70.7 \%$ respectively; $p<0.001)$. Figure 2 outlines the distribution of esophageal motility disorder by subtype, as per classification at the time of data collection. In addition to conditions described according to CCV3.0, basal LES sphincter pressure was recorded and considered abnormal if it was outside the accepted normative values $(13 \mathrm{mmHg}-43 \mathrm{mmHg})$. 
Table 1

Manometry and Timed Barium Esophagram (TBE) in case-cohort and control-cohort. *Please see figure 2 for further elaboration of the classification of abnormal subtypes.

\begin{tabular}{|llll|}
\hline Manometry & Cases & Controls & p-value \\
\hline Number of Patients & 114 & 41 & \\
\hline Age (years); Mean \pm SD & $61.3 \pm 13.0$ & $55.1 \pm 15.4$ & 0.75 \\
\hline BMl; Mean \pm SD & $29.9 \pm 5.5$ & $28.5 \pm 6.7$ & 0.75 \\
\hline Sex, N (\%): & & & 0.01 \\
\hline Male & $47(41.2 \%)$ & $8(19.5 \%)$ & \\
\hline Female & $67(58.8 \%)$ & $33(80.5 \%)$ & \\
\hline Hernia, N (\%) & $34(29.8 \%)$ & $20(48.8 \%)$ & 0.003 \\
\hline Size (cm); Mean \pm SD & $2.9 \pm 1.9$ & $3.0 \pm 1.4$ & 0.89 \\
\hline TBE & Cases & Controls & p-value \\
\hline Total, N & 114 & 41 & \\
\hline Normal & 40 & 29 & $<0.001$ \\
\hline Abnormal* & 74 & 12 & \\
\hline Tablet Arrest, N (\%) & $41(36.0 \%)$ & $4(9.8 \%)$ & $<0.001$ \\
\hline Hiatal Hernia, N (\%) & $5(4.4 \%)$ & $4(9.8 \%)$ & $<0.001$ \\
\hline Stricture, N (\%) & $3(2.6 \%)$ & $0(0 \%)$ & - \\
\hline TBE 1' N & $54(48.2 \%)$ & $10(24.4 \%)$ & $\mathbf{0 . 0 0 3}$ \\
\hline Column > 0 mm, N(\%) & & & 0.43925 \\
\hline Mean \pm SD (mm) & $53(73.9 \%)$ & $27(59.4 \%)$ & $<.001$ \\
\hline TBE 5' N & $37(32.7 \%)$ & $2(4.9 \%)$ & \\
\hline Column > 0 mm, N(\%) & & & \\
\hline Mean \pm SD (mm) & $26.3(49.3)$ & $2.4(13.1 \%)$ & 0.44 \\
\hline & & & \\
\hline
\end{tabular}

Table 2: Manometry Data. Normal HRM on TBE was defined as column height $<17 \mathrm{mmHg}$, as measured from gastroesophageal junction to the height of the top of the column 


\begin{tabular}{|c|c|c|c|c|}
\hline Manometry Data & & Cases & Controls & p-value \\
\hline Number $(\mathrm{N})$ & & 114 & 41 & \\
\hline \multirow[t]{4}{*}{ 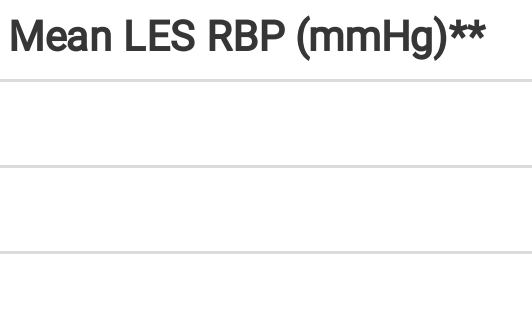 } & 0.19 & & & \\
\hline & Normal, N (\%) & $53(46.5 \%)$ & $24(58.5 \%)$ & \\
\hline & Abnormal, N (\%) & $61(53.5 \%)$ & $17(41.5 \%)$ & \\
\hline & Mean \pm SD & $45.7(21.2 \%)$ & $34.5 \pm 17.1$ & 0.48 \\
\hline \multirow[t]{4}{*}{ Mean LES RP $(\mathrm{mmHg})^{\wedge}$} & $<0.001$ & & & \\
\hline & Normal, N (\%) & $23(20.2 \%)$ & $23(56.1 \%)$ & \\
\hline & Abnormal, N (\%) & $91(79.8 \%)$ & $18(43.9 \%)$ & \\
\hline & Mean \pm SD & $22.3 \pm 11.0$ & $13.3 \pm 7.5$ & 0.48 \\
\hline \multirow[t]{4}{*}{$\mathrm{DCl}(\mathrm{mmHg}-\mathrm{cm}-\mathrm{s})^{\circ}$} & 0.05 & & & \\
\hline & Normal, N (\%) & $76(66.7 \%)$ & $34(82.9 \%)$ & \\
\hline & Abnormal, N (\%) & $38(33.3 \%)$ & $7(17.1 \%)$ & \\
\hline & Mean \pm SD & $3708.8 \pm 3952.9$ & $2116.5 \pm 2079.3$ & 0.33 \\
\hline \multirow[t]{4}{*}{ LES IBP $(\mathrm{mmHg})^{x}$} & $<0.001$ & & & \\
\hline & Normal, N (\%) & $55(48.2 \%)$ & $35(85.4 \%)$ & \\
\hline & Abnormal, N (\%) & $59(51.8 \%)$ & $6(14.6 \%)$ & \\
\hline & Mean \pm SD & $7.4 \pm 7.1$ & $3.0 \pm 4.6$ & 0.44 \\
\hline \multirow[t]{4}{*}{ LES AVG MAX $(\mathrm{mmHg})^{\mathrm{z}}$} & 0.95 & & & \\
\hline & Normal, N (\%) & $\mathrm{N} / \mathrm{A}$ & 41 & \\
\hline & Abnormal, N (\%) & 114 & $\mathrm{~N} / \mathrm{A}$ & \\
\hline & Mean \pm SD & $22.6 \pm 12.4$ & $12.7 \pm 3.1$ & \\
\hline \multirow[t]{3}{*}{ Incomplete bolus clearance } & & & & 0.002 \\
\hline & Normal, N (\%) & $49(43.4 \%)$ & $29(70.7 \%)$ & \\
\hline & Abnormal, N (\%) & $64(56.6 \%)$ & 12 (29.3\%) & \\
\hline Manometry & & & & $<0.001$ \\
\hline
\end{tabular}

*BMI: body mass index; ${ }^{\star \star}$ mean lower esophageal sphincter respiratory basal pressure; ${ }^{\wedge}$ mean lower esophageal sphincter residual pressure; ${ }^{\circ}$ distal contractile integral; ${ }^{x}$ lower esophageal sphincter intrabolus pressure; ${ }^{\mathrm{z}}$ average maximum lower esophageal sphincter pressure in $\mathrm{mmHg}$ 


\begin{tabular}{|c|c|c|c|}
\hline Manometry Data & Cases & Controls & p-value \\
\hline Normal, N (\%) & $5(4.4 \%)$ & $12(29.3 \%)$ & \\
\hline Abnormal, N (\%) & 109 (95.6\%) & $29(70.7 \%)$ & \\
\hline \multicolumn{4}{|c|}{$\begin{array}{l}\text { *BMI: body mass index; }{ }^{* \star} \text { mean lower esophageal sphincter respiratory basal pressure; }{ }^{\wedge} \text { mean lower } \\
\text { esophageal sphincter residual pressure; }{ }^{\circ} \text { distal contractile integral; }{ }^{x} \text { lower esophageal sphincter } \\
\text { intrabolus pressure; }{ }^{\mathrm{z}} \text { average maximum lower esophageal sphincter pressure in } \mathrm{mmHg}\end{array}$} \\
\hline
\end{tabular}

\section{Timed Barium Esophagram}

Case-patients were found to have significantly more abnormalities reported on TBE than control-patients ( $64.9 \%$ vs. $29.1 \%$ respectively; $p<0.001$ ). As outlined in Table 1, a significant difference remained between the two groups in the presence of hiatal hernias $(p<0.001)$. There was a higher frequency of patients with retention of the barium tablet in the case group compared to the control group $(p<0.001)$. A higher proportion of case-patients than control-patients had retention of liquid barium at one minute ( $48.2 \%$ vs. $24.4 \% ; p=0.003)$. More case-patients exhibited barium retention at five minutes compared to controlpatients $(32.7 \%$ vs. $4.9 \%$; $p<0.001)$. Additionally, AM-IBP was significantly related to tablet arrest $(p=0.011)$ in a logistic regression model, which correctly predicted tablet arrest in $64 \%$ of patients. An optimal prediction point, found by maximizing Youden's index, was associated with an average max IBP of $20.1 \mathrm{~mm} \mathrm{H}$ (Figure 3). Of those with tablet arrest, $69 \%$ had an average maximum IBP $\geq 20.1 \mathrm{~mm} \mathrm{Hg}$. We noted no differences of significance in the quantitative measurements of the column heights amongst the two groups.

A backward elimination procedure evaluating EGJOO subsets resulted in a model revealing abnormal TBE is more often associated with both tablet arrest and Types I-III achalasia. Specifically, patients with abnormal TBE were found to have a $62 \%$ higher likelihood for TBE tablet retention compared to those with normal TBE $(p<0.001)$.

\section{High-resolution manometry}

A higher percentage of case-patients vs. control-patients had abnormal manometries ( $95.6 \%$ vs. $70.7 \%$ respectively; $\mathrm{p}<0.001)$. The proportion of abnormal manometric findings were significantly different between the two groups in mean lower esophageal sphincter respiratory basal pressure, mean average lower esophageal sphincter residual pressure, distal contractile integral, intrabolus pressure at the lower esophageal sphincter, and incomplete bolus clearance (Table 2). We also found significance with a higher frequency of tablet retention in the case group than in the control group ( $88.8 \%$ vs. $64.9 \%$ respectively; $\mathrm{p}=0.015)$. A hiatal hernia was present in more people in the control group than in the case group (48.8\% vs. $29.8 \%$ respectively; $p=0.003)$.

\section{Discussion}


Our significant findings were AM-IBP correlated with tablet arrest and liquid bolus retention, which maintained significance by logistic regression. Specifically, an optimal prediction point for AM-IBP was found at $20.1 \mathrm{~mm} \mathrm{Hg}$. Additionally, we found that both anatomical and clinically relevant EGJOO patients exhibit elevated IBP manometrically. EGJOO is a heterogeneous collection of esophageal dysmotility types with manometry characterization crucial in the diagnosis. In the updated CCv.4, clinical correlation merits higher consideration to allow for improved prognostication of clinical outcomes. In what we have found to be the largest cohort of patients with elevated IBP, this association can help diagnose clinically relevant esophageal obstructive disorders, particularly in the context of the updated CCv4.0 metrics recognizing the utility of IBP.

As a marker of flow impedance within the esophagus, AM-IBP proves to be a novel and objective metric in grading the severity of other conditions known to cause strictures or similar changes to the functionality of the esophagus. ${ }^{19}$ When peristalsis is present, IBP can be an indirect measure of the distensibility of the distal esophagus or the EGJ. ${ }^{18,21-23}$ The AM-IBP measurement recorded by HRM demonstrates the compartmentalized force exerted on a bolus during esophageal peristalsis.,16-18 Our study is consistent with findings previously reported for elevated IBP in EGJOO. ${ }^{24-28}$ For example, a survey by Scherer et al. examined all the composite characteristics of patients with post-Nissen fundoplication $(n=8)$ and patients with functional obstruction $(n=16)$ and found a higher frequency of maximum IBP (defined as IBP $>30 \mathrm{mmHg}$ ) in patients with post-Nissen fundoplication when compared to patients with functional obstruction ( $n=63$ swallows; $79 \%$ vs. $n=86$ swallows; $54 \%$, respectively). ${ }^{30}$

Continued refinement in diagnostics has led to improved metrics to allow for a reliable and reproducible evaluation of esophageal motility. With the advent of HRM, additional information about obstructive forces near the EGJ beyond conventional manometry is available due to the number and spacing of solidstate pressure sensors in HRM. ${ }^{9,12,17,27}$ This increased number of sensors in HRM essentially eliminates a deficiency of conventional manometric systems: the problem of movement-related artifacts. ${ }^{8,28-29}$

CCV4.0 provides a more robust characterization of outflow disorders such as EGJOO in a more patientoriented protocol. 5,6 Maneuvers in various positions in combination with clinical symptoms offer a more specific depiction. Therein, incorporating IBP in diagnosing disorders of EGJOO can allow for more precise diagnosis to mitigate over-diagnosis secondary to artifacts.

There are limitations to our study to be acknowledged. First, we analyzed retrospective data at a single center; thus, results may not reflect other centers. However, consistent with our hypothesis and literature to date, higher intrabolus pressures are commonly found with disorders of outflow obstruction (more minor with different subtypes). ${ }^{34-37}$ Second, since this data gathering, modalities such as endoluminal functional lumen imaging probe (EndoFLIP ${ }^{T M}$ Medtronic Minneapolis, MN 55432) have become more prevalent in diagnosing EGJ diseases; EndoFLIPTM was not performed during this study. ${ }^{38,42}$ The majority of patients referred for HRM had clinical symptoms concerning for non-obstructive dysphagia, which is an inherent selection bias. Finally, despite continual improvements, a limitation to acknowledge is the 
lack of safeguards in the ManoView v.3.0 software metric's susceptibility to erroneous pressure artifact. Nonetheless, our study provides results for evaluating the association between AM-IBP barium retention on timed barium esophagram and how it relates to disorders of outflow obstruction. Our study validates the clinical utility of IBP as an important manometric measure and supports its incorporation in CCv4.0.

\section{Conclusion}

AM-IBP in the interpretation of HRM can be a surrogate marker for resistance and obstruction in the esophagus, particularly in EGJOO subsets. Measuring esophageal stasis is vital for disorders of esophageal gastrojunctional outflow obstruction. An abnormal AM-IBP with an optimal prediction point of $20.1 \mathrm{~mm} \mathrm{Hg}$ was associated with a higher probability of retaining liquid bolus or barium tablet arrest on TBE and esophageal dysmotility on HRM. Future studies can further characterize how elevated intrabolus pressure correlates to poor bolus transit in the classification of esophageal dysmotility.

\section{Declarations}

Ethics approval and consent to participate:

All methods were carried out in accordance with relevant guidelines and regulations. All experimental protocols were approved by a named institutional and/or licensing committee. Informed consent was obtained from all subjects and/or their legal guardian(s). IRB approval IRB00053297 granted per Wake Forest Institutional Review Board in Winston-Salem, North Carolina USA.

\section{Consent for publication:}

Not applicable.

\section{Availability of data and materials:}

The datasets generated and/or analyzed during the current study are not publicly available due hospital patient confidentiality restrictions due to potentially identifying information, but are available from the corresponding author on reasonable request.

\section{Competing interests:}

None of the authors have any potential financial or non-financial conflicts to disclose.

\section{Funding:}

Wake Forest Baptist hospital has assisted in funding for this research study.

Authors' contributions: 
SC: Project concept/design; data collection and analysis; critical revision for important intellectual content; approved final draft

SS: Project concept/design; data collection and analysis; approved final draft

JE: Project concept/design; assisted in data analysis and execution; critical revision for important intellectual content; approved final draft

KM: Project concept/design; data analysis; drafting and preparing the manuscript; critical revision for important intellectual content; approved final draft

\section{Acknowledgements:}

We wish to acknowledge Joni Evans for her assistance in analyzing this data, Steven Clayton for his mentorship, and Wake Forest Baptist hospital for funding support.

\section{References}

1. Lin Z, Kahrilas PJ, Roman S, et al. Refining the criterion for an abnormal Integrated Relaxation Pressure in esophageal pressure topography based on the pattern of esophageal contractility using a classification and regression tree model. Neurogastroenterol Motil. 2012;24(8):e356-e363.

2. Weijenborg PW, Kessing BF, Smout AJ, et al. Normal values for solid-state esophageal high-resolution manometry in a European population; an overview of all current metrics. Neurogastroenterol Motil. 2014.

3. Clayton S, Patel R, Richter J. Functional and Anatomic Esophagogastric Junction Outflow Obstruction: Manometry, Timed Barium Esophagram Findings, and Treatment Outcomes. Clin Gastroenterol Hepatol. 2016;14(6):907-911.

4. Yadlapati R, Pandolfino J, Fox M, et al. What is new in Chicago Classification version 4.0? Neurogastroenterol Motil. 2021 Jan;33(1):e14053. doi: 10.1111/nmo.14053.

5. Richter J, Clayton S. Diagnosis and Management of Esophagogastric Junction Outflow Obstruction. Am J Gastroenterol. 2019;114(4):544-547.

6. Yadlapati R. High-resolution esophageal manometry: interpretation in clinical practice. Curr Opin Gastroenterol. 2017;33(4):301-309. doi:10.1097/MOG.0000000000000369.

7. Ghosh S, Janiak P, Fox M, et al. Physiology of the esophageal transition zone in the presence of chronic bolus retention: studies using concurrent high-resolution manometry and digital fluoroscopy. Neurogastroenterol Motil. 2008;20:750-759.

8. O'Rourke A, Lazar A, Murphy B, et al. Utility of Esophagram versus High-Resolution Manometry in the Detection of Esophageal Dysmotility. Otolaryngol Head Neck Surg. 2016;154(5):888-91. doi: $10.1177 / 0194599816629379$. 
9. Roman S, Huot L, Zerbib F, et al. High-resolution manometry improves the diagnosis of esophageal motility disorders in patients with dysphagia: a randomized multicenter study. Am J Gastroenterol. 2016;111(3):372-80.

10. Clouse R, Parks T, Haroian L, et al. Development and clinical validation of a solid-state highresolution pressure measurement system for simplified and consistent esophageal manometry. Am J Gastroenterol. 2003;98:S32-S33.

11. Jeong $\mathrm{S}$, Park $\mathrm{M}, \mathrm{Kim} \mathrm{H}$, et al. Utilizing intrabolus pressure and esophagogastric junction pressure to predict transit in patients with Dysphagia. J Neurogastroenterol Motil. 2014;20(1):74-78. doi:10.5056/jnm.2014.20.1.74.

12. Carlson D, Pandolfino J. High-Resolution Manometry in Clinical Practice. Gastroenterol Hepatol (NY). 2015;11(6):374-384.

13. Quader F, Reddy C, Patel A, et al. Elevated intrabolus pressure identifies obstructive processes when integrated relaxation pressure is normal on esophageal high-resolution manometry. Am J of Phys Gastro and Liver Phys. 2017;313:G73-G79.

14. Tamhankar AP, Almogy G, Arain MA, et al. Surgical management of hypertensive lower esophageal sphincter with dysphagia or chest pain. J Gastrointest Surg. 2003;7:990-6.

15. Farrokhi F, Vaezi MF. Idiopathic (primary) achalasia. Orphanet J Rare Dis. 2007; 26(2):38. doi: 10.1186/1750-1172-2-38.

16. Niebisch S, Wilshire C, Peters J. Systematic analysis of esophageal pressure topography in highresolution manometry of 68 normal volunteers. Dis of the esophag: official $\mathrm{J}$ Internat Societ for Dis of the Esophag / ISDE. 2013;26.

17. Cho Y, Lipowska A, Nicodème F, et al. Assessing Bolus Retention in Achalasia using High Resolution Manometry with Impedance: A Comparator Study with Timed Barium Esophagram. The Am J of Gastroent. 2014;109(6):829-835. doi:10.1038/ajg.2014.61.

18. Yadlapati R, Kahrilas P, Fox M, et al. Esophageal motility disorders on high-resolution manometry: Chicago classification version 4.0. Neurogastroenterol Motil. 2021; 33(1), e14058.

19. Fox M, Hebbard G, Janiak P, et al. High-resolution manometry predicts the success of oesophageal bolus transport and identifies clinically important abnormalities not detected by conventional manometry. Neurogastroenterol Motil. 2004;16:533-542.

20. Kessing BF, Weijenborg PW, Smout AJPM, et al. Water-perfused esophageal high-resolution manometry: normal values and validation. Am J Physiol -Gastroint and Liver Physiol. 2014;306:G491-G495.

21. Kahrilas P, Ghosh S, Pandolfino J. Esophageal motility disorders in terms of pressure topography: the Chicago Classification. J Clin Gastroenterol. 2008;42:627-635.

22. Kahrilas PJ, Bredenoord AJ, Fox M, et al. The Chicago Classification of esophageal motility disorders, v3.0. Neurogastroenterol Motil. 2015;27:160-74.

23. Smith J, Clayton S. Elevated Average Maximum Intrabolus Pressure on High Resolution Manometry Is Associated with Esophageal Dysmotility and Delayed Esophageal Emptying on Timed Barium 
Esophagrams: Presidential Poster Award, Am J of Gastroent. 2018;113:182-183.

24. Hoscheit M, Gabbard S. Elevated Intrabolus Pressure Predicts Abnormal Timed Barium Esophagram in Esophagogastric Junction Outflow Obstruction. J Neurogastroenterol Motil. 2019 Oct $30 ; 25(4): 521-524$.

25. Cho YK. Does Elevated Intrabolus Pressure Predict Esophageal Stasis in Dysphagia Patients? J Neurogastroenterol Motil. 2019 Oct 30;25(4):481-482.

26. Rohof WOA, Bredenoord AJ. Chicago Classification of Esophageal Motility Disorders: Lessons Learned. Curr Gastroenterol Rep. 2017;19(8):37. doi:10.1007/s11894-017-0576-7

27. De Schepper HU, Ponds FA, Oors JM, et al. Distal esophageal spasm and the Chicago classification: is timing everything? Neurogastroenterol Motil. 2016;28(2):260-5.

28. Nguyen HN, Silny J, Matern S. Multiple intraluminal electrical impedancometry for recording of upper gastrointestinal motility: current results and further implications. Am J Gastroenterol. 1999;94:30617.

29. Blonski W, Hila A, Vela MF, et al. An analysis of distal esophageal impedance in individuals with and without esophageal motility abnormalities. J Clin Gastroenterol. 2008;42:776-81.

30. Scherer JR, Kwiatek MA, Soper NJ, et al. Functional esophagogastric junction obstruction with intact peristalsis: a heterogeneous syndrome sometimes akin to achalasia. J Gastrointest Surg. 2009;13:2219-25.

31. Singh E, Rife C, Clayton SB, et al. Lessons Learned From High Resolution Manometry - InterObserver Variability in Esophageal Body Measurements Amongst New Physician Users. Gastroenterology. 2011;140:S-225.

32. Vaezi MF, Baker ME, Achkar E, et al. Timed barium oesophagram: better predictor of long term success after pneumatic dilation in achalasia than symptom assessment. Gut. 2002;50:765-70.

33. Vaezi MF, Baker ME, Richter JE. Assessment of esophageal emptying post-pneumatic dilation: use of the timed barium esophagram. Am J Gastroenterol. 1999;94:1802-7.

34. Ren J, Massey BT, Dodds WJ, et al. Determinants of intrabolus pressure during esophageal peristaltic bolus transport. Am J Physiol. 1993;264:G407-13.

35. van Hoeij FB, Smout AJ, Bredenoord AJ. Characterization of idiopathic esophagogastric junction outflow obstruction. Neurogastroenterol Motil. 2015;27(9):1310-6.

36. Mittal R, Vaezi MF. Esophageal Motility Disorders and Gastroesophageal Reflux Disease. New Eng J of Med. 2020;383:1961-1972.

37. Lei W, Vaezi M, Naik R, et al. Mucosal impedance testing: A new diagnostic testing in gastroesophageal reflux disease, J of the Formosan Med Assoc. 2019.

38. Galey KM, Wilshire CL, Niebisch S, et al. Atypical variants of classic achalasia are common and currently under-recognized: a study of prevalence and clinical features. J Am Coll Surg. 2011;213:155-61; discussion 162-3. 
39. Sadowski DC, Ackah F, Jiang B, et al. Achalasia: incidence, prevalence and survival. A populationbased study. Neurogastroenterol Motil. 2010;22:e256-61.

40. Jones B, Clayton S. Mechanical Etiologies Associated with the Diagnosis of Esophageal Outflow Obstruction on High Resolution Manometry. Adv Res Gastroentero Hepatol. 2020;15(1): 555928.

41. Agrawal A, Tutuian R, Hila A, et al. Ingestion of acidic foods mimics gastroesophageal reflux during pH monitoring. Dig Dis Sci. 2005;50:1916-20.

42. Kim GH. Is EndoFLIP Useful for Predicting Clinical Outcomes after Peroral Endoscopic Myotomy in Patients with Achalasia? Gut Liver. 2019;13(1):3-4. doi:10.5009/gnl18443.

\section{Figures}

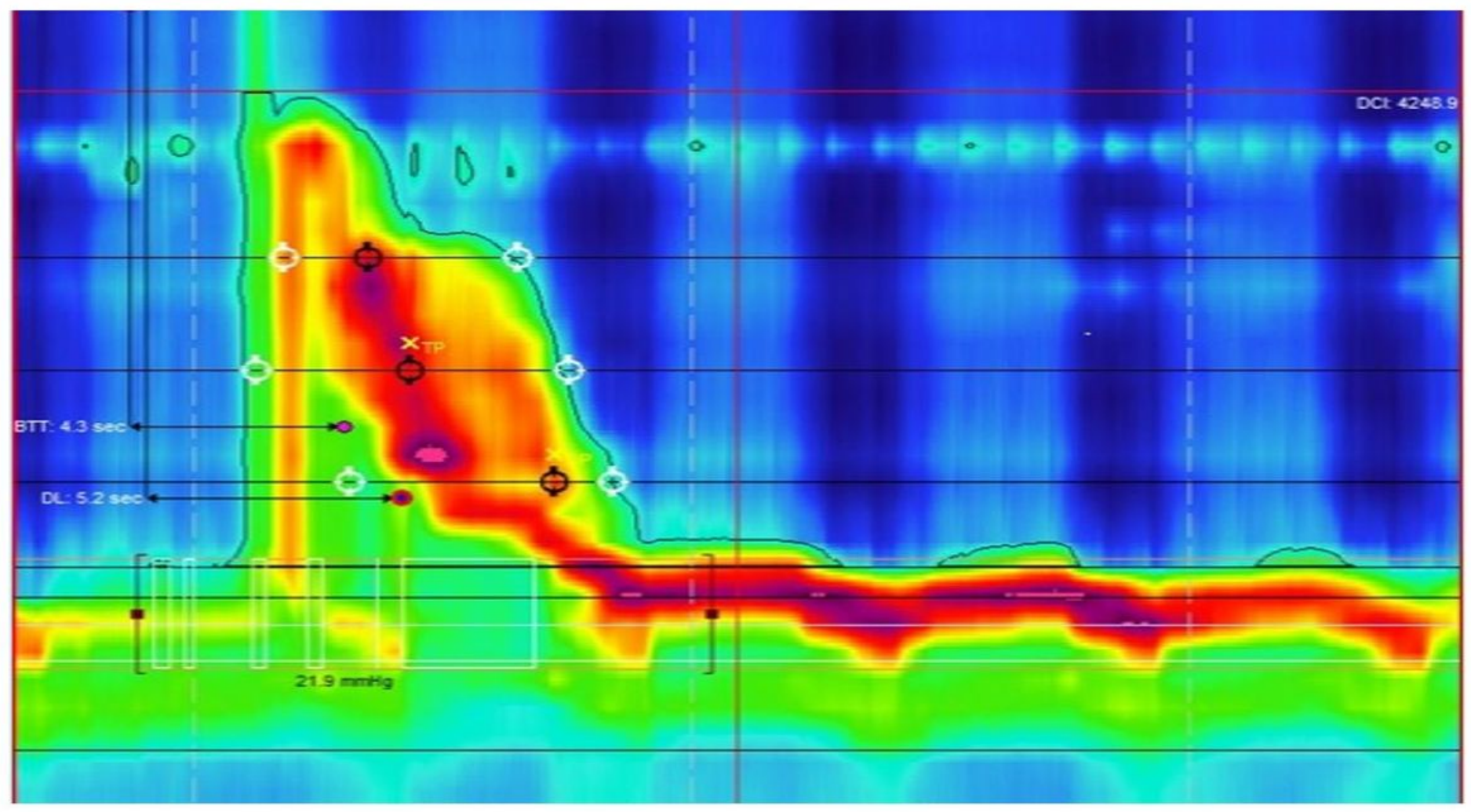

\section{Figure 1}

Patient with esophagogastric outflow obstruction on high-resolution manometry with intrabolus pressure $>27 \mathrm{mmHg}$ 


\section{Types of Esophageal Dysmotility}

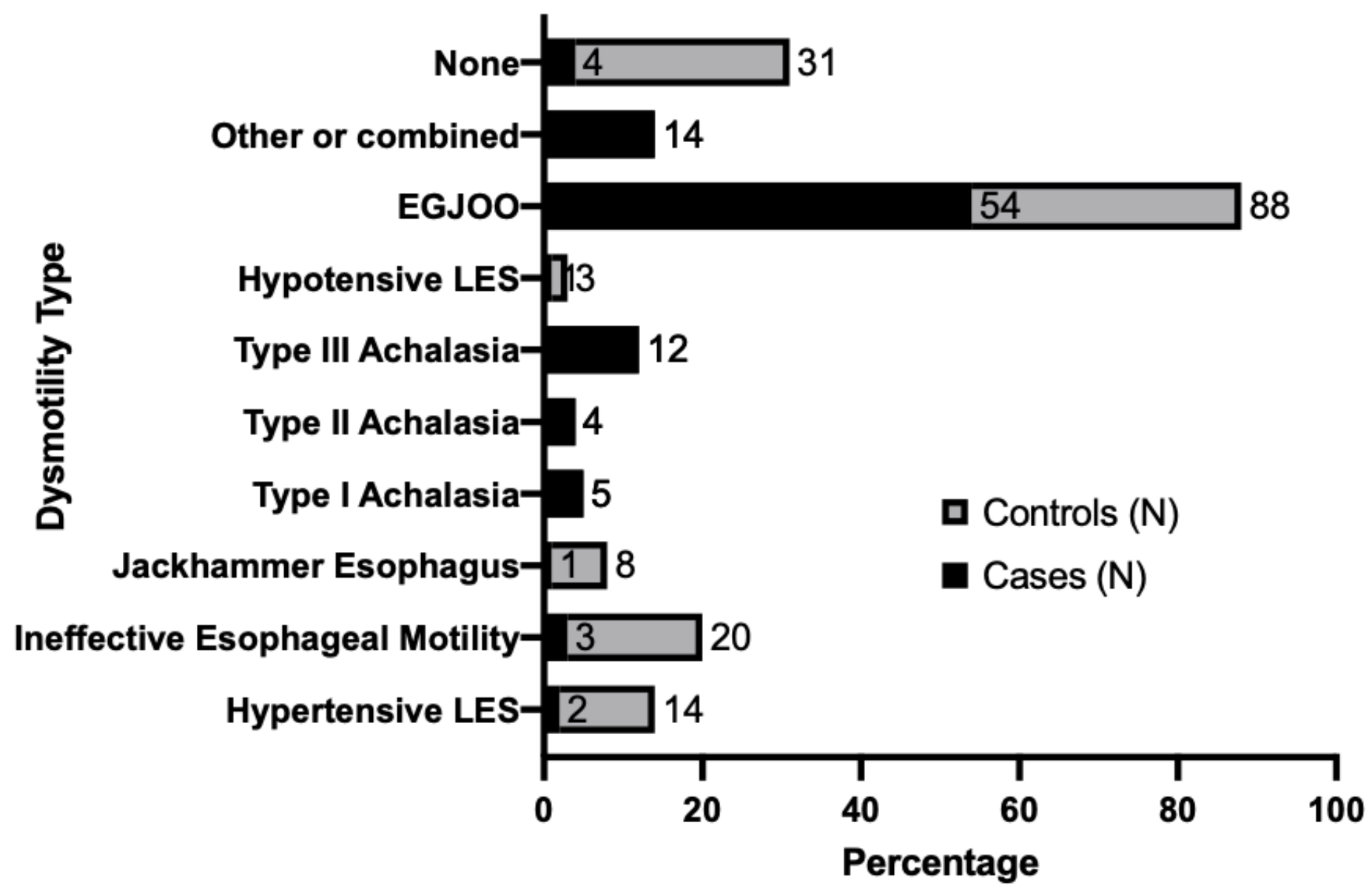

Figure 2

Distribution of esophageal motility disorder by subtype, as consistent with Chicago classification 3.0 at time of data collection. In addition to disorders described according to CCv3.0, basal LES sphincter pressure was recorded and considered abnormal if it was outside the accepted normative values $(13 \mathrm{mmHg}-43 \mathrm{mmHg})$. 


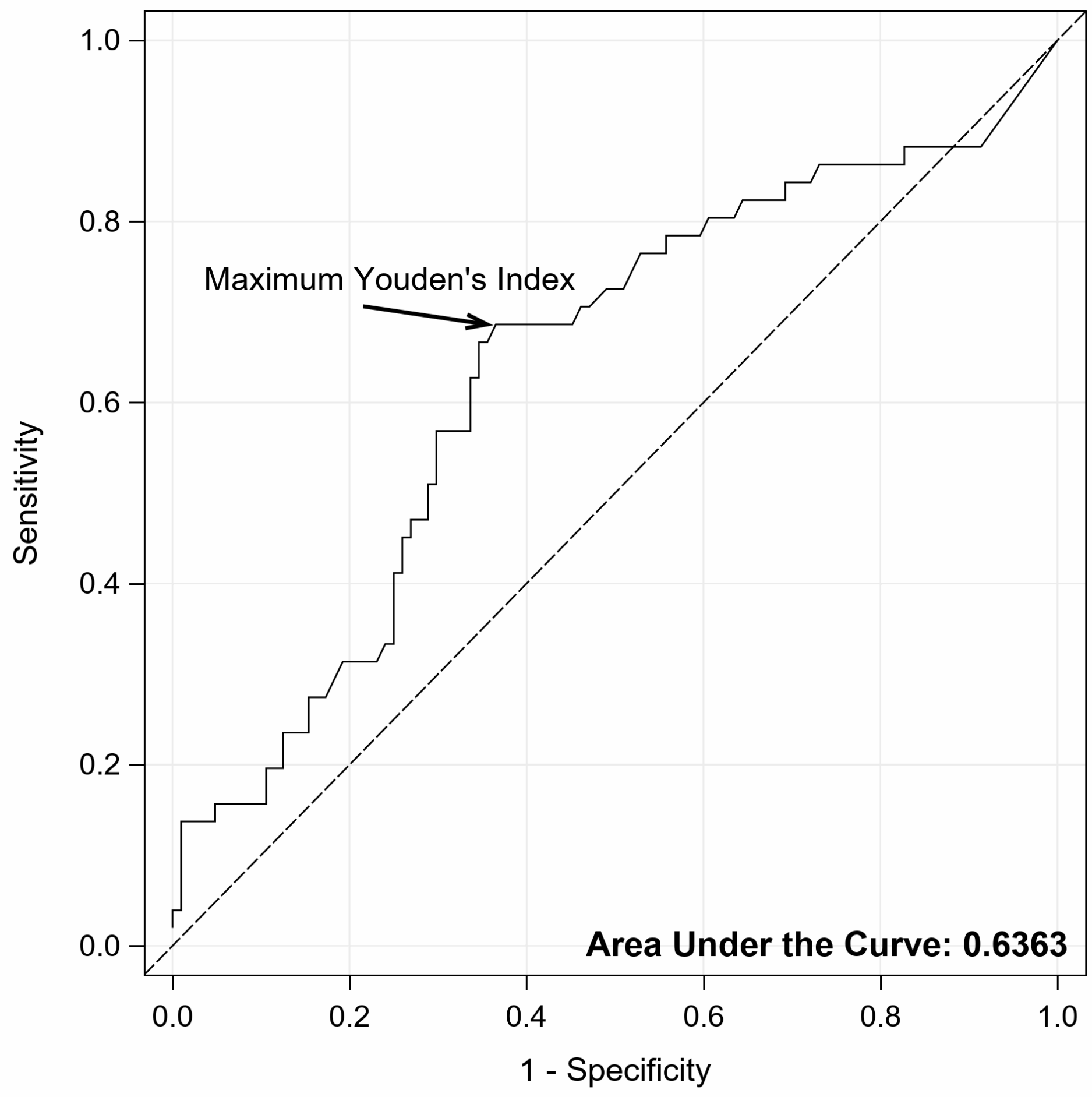

Figure 3

Receiver operating curve for optimal prediction point for tablet arrest at an average max IBP at $20.1 \mathrm{~mm}$ $\mathrm{Hg}$. Additionally, the AUC for AM-IBP, as shown correctly predicted tablet arrest in $64 \%$ of patients. 